\title{
Explosive synchronization in adaptive and multilayer networks
}

\author{
Xiyun Zhang, ${ }^{1}$ Stefano Boccaletti, ${ }^{2,3,-\circledast}$ Shuguang Guan, ${ }^{1}$ and Zonghua Liu ${ }^{1,0}$ \\ ${ }^{I}$ Department of Physics, East China Normal University, Shanghai, 200062, China \\ ${ }^{2}$ CNR-Institute of Complex Systems, Via Madonna del Piano 10, 50019 Sesto Fiorentino, Florence, Italy \\ ${ }^{3}$ The Italian Embassy in Israel, 25 Hamered Street, 68125 Tel Aviv, Israel
}

(Dated: October 14, 2014)

\begin{abstract}
Explosive synchronization (ES) is nowadays a hot topic of interest in nonlinear science and complex networks. So far, it is conjectured that ES is rooted in the setting of specific microscopic correlation features between the natural frequencies of the networked oscillators and their effective coupling strengths. We show that ES, in fact, is far more general, and can occur in adaptive and multilayer networks also in the absence of such correlation properties. Precisely, we first report evidence of ES in the absence of correlation for networks where a fraction $f$ of the nodes have links adaptively controlled by a local order parameter, and then we extend the study to a variety of two-layer networks with a fraction $f$ of their nodes coupled each other by means of dependency links. In this latter case, we even show that ES sets in, regardless of the differences in the frequency distribution and/or in the topology of connections between the two layers. Finally, we provide a rigorous, analytical, treatment to properly ground all the observed scenario, and to facilitate the understanding of the actual mechanisms at the basis of ES in real-world systems.
\end{abstract}

PACS numbers: 89.75.-k, 05.45.Xt

Phase transitions in ensembles of networked systems is one of the hottest topics of interest in current days. Recently, it was pointed out that the transition of an ensemble of networked phase oscillators from incoherence to synchronization can be first-order like, discontinuous and irreversible, called explosive synchronization (ES). This discovery is of huge significance, as abrupt phase transitions are indeed featured by a variety of real world systems [1]: from epileptic seizures in the brain [2], to cascading failure of power grids [3] and jamming in the Internet [4]. Since its finding in 2005 [5], ES has been paid a great attention [6-13]. For instance, it was studied in the context of periodic phase oscillators for scale-free (SF) network's topologies, with an ad-hoc imposed positive correlation between the natural frequencies of the oscillators and the degrees of nodes [6], and the experimental verification of such a setup was given in an electronic circuits with a star configuration and chaotic units [7]. Later on, ES was described for generic network's topologies (either SF or non-SF) in a modified Kuramoto model, with a positive correlation between the natural frequencies of oscillators and their coupling strengths [10, 11]. The two kinds of positive correlations can be unified into the framework of effective couplings in meanfield, which are weighted to be proportional to the frequency (or the frequency difference) of the oscillators [9, 10]. More recently, it was also shown that ES can be considered as the counterpart of an explosive percolation process in dynamical phase space [13].

The accepted state of knowledge on this matter is, hence, that ES has a basic and key microscopic root in the setting of local correlation features (either ad-hoc imposed [6, 7], or spontaneously emerging [8-13]) between the natural frequency of a networked oscillator and its degree, or effective coupling strength. In this Letter, we fundamentally revisit the issue, and provide an answer to the following question: Is it possible to observe ES in networked oscillators without the presence of any kind of microscopic correlation features? We first consider a network where the coupling of a fraction $f$ of the nodes is adaptively controlled by a local order parameter, and show that ES emerges, indeed, when the value of $f$ is over a critical value $f_{c}$. We then extend the study of ES to multilayer networks. Precisely, we give evidence that ES is a generic feature of two-layered networks, when a fraction $f$ of their nodes are coupled with each other by means of $d e$ pendency links, i.e. when the coupling strength of a node in a layer is adaptively controlled by the local order parameter of the corresponding node in the other layer. Further, we present a rigorous theoretical analysis of mean-field to account for all the described scenario. Finally, we formulate the main conclusion: the robustness of our findings suggests that all previous studies on ES can be, in fact, unified into a common root, that of suppressing the formation of giant clusters.

Let us begin with considering a network of $N$ Kuramotolike phase oscillators, with an explicit fraction $f$ of the nodes adaptively controlled by a local order parameter. In our model, the evolution of each oscillator is ruled by

$$
\dot{\theta}_{i}=\omega_{i}+\lambda \alpha_{i} \sum_{j=1}^{N} A_{i j} \sin \left(\theta_{j}-\theta_{i}\right)
$$

where $i=1, \cdots, N, \omega_{i}\left(\theta_{i}\right)$ is the natural frequency (the instantaneous phase) of the $i^{t h}$ oscillator, $\lambda$ is the overall coupling strength, $k_{i}=\sum_{j=1}^{N} A_{i j}$ is the degree of node $i$, and $A_{i j}$ are the elements of the the network's adjacency matrix $A$ ( $A_{i j}=1$ when the nodes $i$ and $j$ are connected, and $A_{i j}=0$ otherwise). When compared to previous studies, the key feature of Eq. (1) is the presence of the parameter $\alpha_{i}$. To define $\alpha_{i}$, we introduce the instantaneous local order parameter for the $i^{\text {th }}$ oscillator of the network, as

$$
r_{i}(t) e^{i \phi}=\frac{1}{k_{i}} \sum_{j=1}^{k_{i}} e^{i \theta_{j}} .
$$




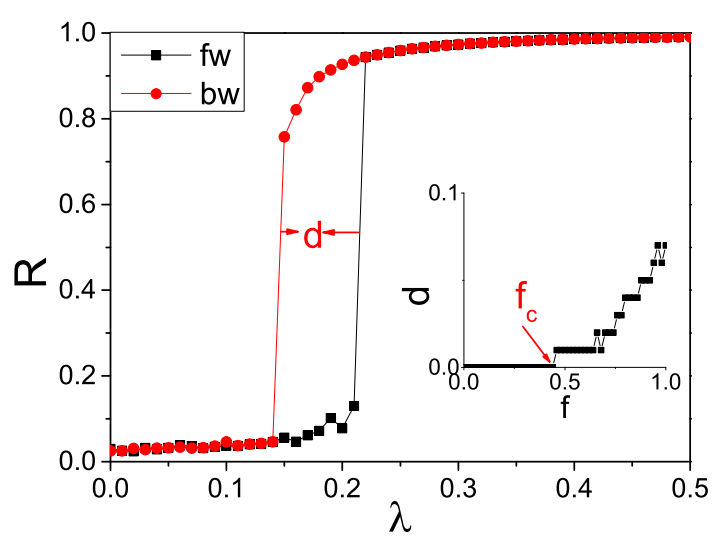

FIG. 1: (color online). Forward (black line with squares) and backward (red line with circles) synchronization transitions for a single network with $N=1,000$ and $f=1$. The inset reports the dependence of $d$ on $f$. See text for the specifications on the network topology and on the frequency distribution.

By definition, $0 \leq r_{i} \leq 1$, and $\phi$ denotes the average (over the ensemble of neighbors) phase. Then, we randomly choose a fraction $f$ of network's nodes, and set for all of them $\alpha_{i}=$ $r_{i}$. The remaining fraction $1-f$ of nodes will have instead, $\alpha_{i}=1$. That is, the fraction $f$ of nodes is actually adaptively controlled by the corresponding local order parameters.

The degree of phase coherence in the network can be measured by means of the global order parameter $R$ defined by

$$
R e^{i \Psi}=\frac{1}{N} \sum_{j=1}^{N} e^{i \theta_{j}}
$$

where $0 \leq R \leq 1$ and $\Psi$ denotes the average phase. In these first numerical simulations, we draw the set of frequencies $\left\{\omega_{i}\right\}$ in Eq. (1) from a random homogeneous distribution $g(\omega)$ in the range $[-1,1]$, and we consider a random ErdôsRényi (ER) network [15] with size $N=1,000$ and average degree $\langle k\rangle=\frac{1}{N} \sum_{i=1}^{N} k_{i}=12$. We increase (decrease) the coupling strength $\lambda$ adiabatically with an increment (decrement) $\delta \lambda=0.01$ from $\lambda=0(\lambda=0.5)$ and compute the stationary value of $R$ for each $\lambda$ [14] during the forward (backward) transition from the incoherent to the phase synchronized state. Fig. 1 reports $R$ vs. $\lambda$ for the case of $f=1$, with the "squares" and "circles" labeling the curves of the forward and backward transitions, respectively. It is evident the presence of an abrupt transition with an associated hysteretic loop in $R$, indicating the occurrence of ES in Eq. (1). Denoting by $d$ the width of such an hysteretic loop, the inset of Fig. 1 reports the dependence of $d$ on $f$, and shows the existence of a critical value $f_{c}$ where $d$ passes from being zero (i.e. a second-order phase transition) to a finite value.

Our second step is showing the generality of our findings for two-layered networks, with different topological and frequency configurations. To this purpose, we construct two independent networks (I and II) and let them have the same size
$N$ in such a way that the nodes on the two layers have a oneto-one correspondence (in the following we denote, for simplicity, each pairs of nodes by the same index $i$ ). We again assume that a fraction $f$ of the nodes between the two networks are coupled with each other by forming dependency links [16]. The equations of motion can be written as

$$
\begin{aligned}
& \dot{\theta}_{i, 1}=\omega_{i, 1}+\lambda \alpha_{i, 1} \sum_{j=1}^{k_{i, 1}} \sin \left(\theta_{j, 1}-\theta_{i, 1}\right), \\
& \dot{\theta}_{i, 2}=\omega_{i, 2}+\lambda \alpha_{i, 2} \sum_{j=1}^{k_{i, 2}} \sin \left(\theta_{j, 2}-\theta_{i, 2}\right),
\end{aligned}
$$

where $i=1, \cdots, N$ and the subscripts 1,2 stand for the layers I and II, respectively. In Eqs. (4), the average degree is $\left\langle k_{1}\right\rangle=\frac{1}{N} \sum_{i=1}^{N} k_{i, 1}\left(\left\langle k_{2}\right\rangle=\frac{1}{N} \sum_{i=1}^{N} k_{i, 2}\right)$ for the layer I (II), and the parameters $\alpha_{i, 1}$ and $\alpha_{i, 2}$ account for the coupling between the two layers. Precisely, we set $\alpha_{i, 1}=r_{i, 2}$ and $\alpha_{i, 2}=r_{i, 1}$ if the pair of nodes $i$ is part of the fraction $f$ of coupled nodes (otherwise we set $\alpha_{i, 1}=\alpha_{i, 2}=1$ ), where $r_{i, 1}$ and $r_{i, 2}$ are defined by $r_{i, 1} e^{i \phi_{1}}=\frac{1}{k_{i, 1}} \sum_{j=1}^{k_{i, 1}} e^{i \theta_{j, 1}}$ and $r_{i, 2} e^{i \phi_{2}}=\frac{1}{k_{i, 2}} \sum_{j=1}^{k_{i, 2}} e^{i \theta_{j, 2}}$. In this way, a group of oscillators in layer $\mathrm{I}$ is adaptively controlled by the local order parameters of the corresponding nodes on layer II, and vice-versa.

Let $R_{1}$ and $R_{2}$ be the global order parameters of the layer I and II, respectively, defined by $R_{1} e^{i \Psi_{1}}=\frac{1}{N} \sum_{j=1}^{N} e^{i \theta_{j, 1}}$ and $R_{2} e^{i \Psi_{2}}=\frac{1}{N} \sum_{j=1}^{N} e^{i \theta_{j, 2}}$. In our simulations, $N=1,000$ and, for convenience, layer I is fixed as a random ER network with average degree $\left\langle k_{1}\right\rangle=12[15]$, and we draw the set frequencies $\left\{\omega_{i, 1}\right\}$ from a random homogeneous distribution in the range $[-1,1]$. Instead, in the following, we will vary both the topology and the frequency distribution $g\left(\omega_{i, 2}\right)$ characterizing layer II. First, we let it be an independent random ER network with the same average degree $\left\langle k_{2}\right\rangle=12$, and we let its frequencies $\left\{\omega_{i, 2}\right\}$ be drawn from an independent random homogeneous distribution in the range $[-1,1]$. Fig. 2 2 a) shows the dependence of $R_{1}$ ("squares" and "triangles") and $R_{2}$ ("circles" and "stars") on $\lambda$ for the case of $f=1$, where the "squares" and "circles" ("triangles" and "stars") denote the forward (backward) transition. One clearly see that ES sets in both layers. Once again, the inset of Fig. 2(a) shows the dependence of $d$ on $f$, and, in analogy with Fig. 11 indicates the presence of a critical value $f_{c}$ for the setting of an irreversible, hysteretic transition.

Second, we let the average degree $\left\langle k_{2}\right\rangle$ change from 12 to 6 while keeping all other parameters unchanged. The results for $f=1$ are now shown in Fig. 2(b), with the inset reporting again the dependence of $d$ on $f$. Comparing Fig. 2 (b) with (a), one see that once again ES sets in, though the associated values of $\mathrm{d}$ and $f_{c}$ are, respectively, smaller and larger.

As a third step, we now let the frequency distribution $g\left(\omega_{i, 2}\right)$ change from a homogeneous to a Lorentzian distribution $g(\omega)=\frac{1}{\pi}\left[\frac{\gamma}{\left(\omega-\omega_{0}\right)^{2}+\gamma^{2}}\right]$ with central frequency $\omega_{0}=0$ and $\gamma$ (the half width at half maximum [17]) equal to 0.5, while keeping all other parameters as those of Fig. 2 (a). A 

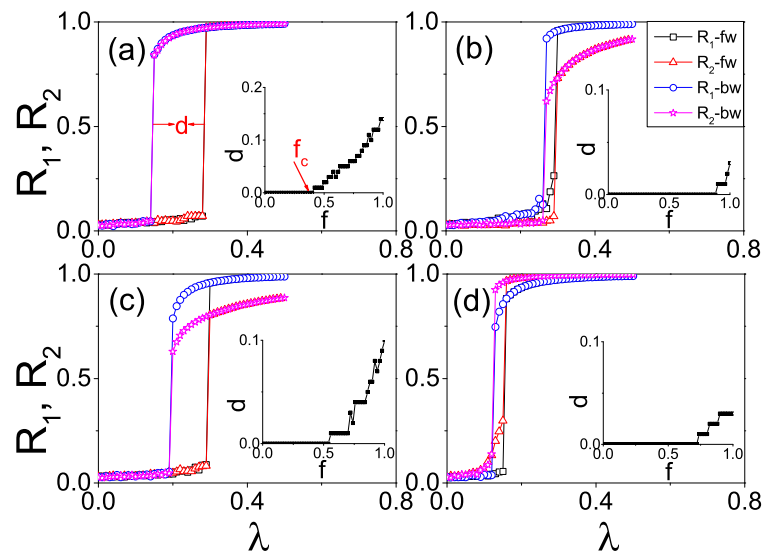

FIG. 2: (color online). Synchronization transitions in two-layer networks for $N=1,000$ and $f=1$. In all plots, "squares" and "circles" ("triangles" and "stars") are used for reporting the values of $R_{1}\left(R_{2}\right)$, ad the insets show the corresponding dependence of $d$ on $f$. Layer I is fixed as a random ER network with average degree $\left\langle k_{1}\right\rangle=12$, and having a random homogeneous distributions of frequencies in the range $[-1,1]$. Layer II has different specifications as follows: (a) it is another ER network with $\left\langle k_{2}\right\rangle=12$, and $g\left(\omega_{i, 2}\right)$ is a random homogeneous distribution in the range $[-1,1]$; (b) it is an ER network with $\left\langle k_{2}\right\rangle=6$ and $g\left(\omega_{i, 2}\right)$ is the same as in (a); (c) it is the same as in (a) but $g\left(\omega_{i, 2}\right)$ is now a Lorentzian distribution (see text for definition) with $\omega_{0}=0$ and $\gamma=0.5$; (d) it is a BA network with $\left\langle k_{2}\right\rangle=12$ and $g\left(\omega_{i, 2}\right)$ is the same as in (a).

significant difference between the homogeneous distribution and the Lorentzian distribution is that the former is homogeneous for every $\omega$ while the latter is heterogeneous with an approximate power law on $\omega$. Nonetheless, Fig. 2/c) (corresponding to $f=1$ ) and its inset clearly indicate the setting of ES.

Finally, we even change the topology of layer II from an ER network to a Barabási-Albert (BA) network [15], while keeping all other parameters as in the case of Fig. 2(a). Notice that, in this latter situation, the topologies of the two layers are essentially different. Once again, the results (reported in Fig. 2(d)) are similar to those of the three panels (a)-(c), and demonstrate the existence of both an hysteretic loop and a critical $f_{c}$ associated with the transition to synchronization.

We now stop for a moment, and try to recall (at this stage) the remarkable conclusions that can be drawn from what already reported so far. ES is a generic property of adaptive networks, as well as multilayer networks, as far as the coupling form used in Eq. (1) and Eq. (4) is taken into account. This has two main implications: (i) it sharply contrasts to previous conclusions that a positive correlation between the natural frequencies of oscillators and their effective couplings is a necessary condition for ES; and (ii) the passage from a firstto a second-order transition is here actually controlled by the coupled fraction $f$ of nodes for which adaptation is effective. Let, therefore, us to speculate more on the correlation issue. The fraction $1-f$ of oscillators not affected by the adap-

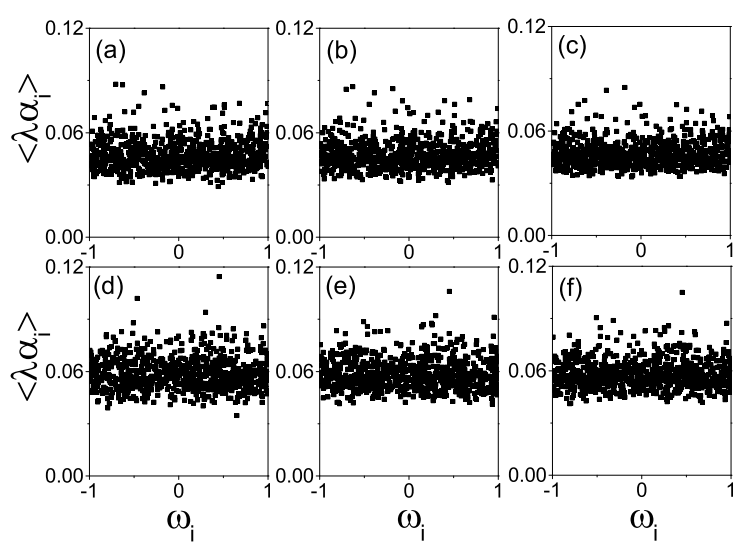

FIG. 3: Correlation properties just before the forward transition to synchronization. Panels (a),(b) and (c) refer to the single network case. They corresponds to $f=0.8, \lambda=0.16$ (i.e. at the forward transition point to synchronization), a single ER network with 1, 000 nodes, $\langle k\rangle=12$, and a uniform frequency distribution in the range $[-1 ; 1]$. Panels (a),(b) and (c) report the average of $\lambda \alpha_{i}$ vs. $\omega_{i}$ over 5,000, 10,000 and 50,000 time steps, respectively. Panels (d),(e) and (f) account, instead for the two-layer case, with $N=1,000$, $f=0.8, \lambda=0.2$ (once again at the forward transition point), and all other parameters as in the caption of Fig. (2)(a)). These latter panels report actually the average of $\lambda \alpha_{i}$ vs. $\omega_{i}$ over 5,000, 10,000 and 50,000 time steps, respectively. Exactly the same qualitative scenario (not shown) occur for the nodes in layer II).

tation mechanism is, by definition and construction, not displaying any form of correlation between nodes' frequencies and coupling strength. As for the faction $f$ of nodes which are, instead, affected by the adaptation rule, it is determinant to notice that the form used in Eq. (2) is actually fully independent on the actual instantaneous phase of the considered oscillators (it, indeed, depends only and solely on the degree of synchronization of the neighboring oscillators of each of these nodes). And, indeed, Fig. 3 show that no correlation features at all between the oscillators' frequencies and their coupling strengths are associated (at the onset of ES in both single-layer and two-layers networks) neither in their short-, nor in their intermediate-, nor even in their long-time averages (as compared with the time scale of their evolution).

In order to gather further information on the overall scenario, we move to some theoretical analysis, and take the case of Fig. 11 with $f=1$ as an example. Substituting Eq. (2) into Eq. (1) one has

$$
\dot{\theta}_{i}=\omega_{i}+\lambda r_{i}^{2} k_{i} \sin \left(\Psi-\theta_{i}\right),
$$

where $\dot{\Psi}=\Omega$ is the group angular velocity. In the mean-field framework, $r_{i}=R$. Letting $\Delta \theta_{i}=\theta_{i}-\Psi$, Eq. (5) becomes

$$
\Delta \dot{\theta}_{i}=\omega_{i}-\Omega-\lambda R^{2} k_{i} \sin \left(\Delta \theta_{i}\right)
$$

If $\left|\omega_{i}-\Omega\right|<\lambda R^{2} k_{i}$, then $\Delta \dot{\theta}_{i}$ reaches a fixed point defined by $\sin \left(\Delta \theta_{i}\right)=\left(\omega_{i}-\Omega\right) / \lambda R^{2} k_{i}$, indicating that the oscillator 
$i$ becomes phase locked to the mean-field. Otherwise, $\Delta \dot{\theta}_{i}$ never reaches a fixed point, indicating that oscillator $i$ drifts at all time. Considering that the natural frequency distribution $g\left(\omega_{i}\right)$ is here symmetric, we have that the average frequency $\Omega=0$. Thus, for the phase-locked oscillators, one has

$$
\Delta \theta_{i}=\arcsin \left(\frac{\omega_{i}}{\lambda R^{2} k_{i}}\right), \quad\left|\omega_{i}\right| \leq \lambda R^{2} k_{i} .
$$

Based on Eq. (7), one can calculate the order parameter $R$. Noticing that $R e^{i \Psi}=\frac{1}{N} \sum_{j=1}^{N} e^{i \theta_{j}}=\frac{1}{N\langle k\rangle} \sum_{j=1}^{N} k_{j} e^{i \theta_{j}}$ and that the drifting oscillators do not contribute to $R$ [8, 18], one has

$$
R=\frac{1}{N\langle k\rangle} \sum_{\left|\omega_{j}\right| \leq \lambda R^{2} k_{j}} k_{j} \cos \left(\Delta \theta_{j}\right) .
$$

Substituting Eq. (7) into Eq. (8) one obtains

$$
R=\frac{1}{N\langle k\rangle} \sum_{\left|\omega_{j}\right| \leq \lambda R^{2} k_{j}} k_{j} \sqrt{1-\left(\frac{\omega_{j}}{\lambda R^{2} k_{j}}\right)^{2}} .
$$

Replacing the summation over degrees by an integration, the contribution of the locked oscillators to the order parameter in the thermodynamic limit is

$$
R=\frac{1}{\langle k\rangle} \int_{|\omega| \leq \lambda R^{2} k} h(k, \omega) k \sqrt{1-\left(\frac{\omega}{\lambda R^{2} k}\right)^{2}} d \omega d k,
$$

where $h(k, \omega)$ is the joint distribution and can be written as $h(k, \omega)=P(k) g(\omega)$ with $P(k)$ being the degree distribution of the network.

If repeated for the case of Eqs. (4), the same treatment yields

$$
\begin{aligned}
& R_{1}=\frac{1}{\left\langle k_{1}\right\rangle} \int_{C_{1}} h\left(k_{1}, \omega_{1}\right) k_{1} \sqrt{1-\left(\frac{\omega_{1}}{\lambda R_{1} R_{2} k_{1}}\right)^{2}} d \omega_{1} d k_{1} \\
& R_{2}=\frac{1}{\left\langle k_{2}\right\rangle} \int_{C_{2}} h\left(k_{2}, \omega_{2}\right) k_{2} \sqrt{1-\left(\frac{\omega_{2}}{\lambda R_{1} R_{2} k_{2}}\right)^{2}} d \omega_{2} d k_{2}(1)
\end{aligned}
$$

where $C_{1,2} \equiv\left|\omega_{1,2}\right| \leq \lambda R_{1} R_{2} k_{1,2}$ are the integration domains, $h\left(k_{1}, \omega_{1}\right)=P\left(k_{1}\right) g\left(\omega_{1}\right)$ and $h\left(k_{2}, \omega_{2}\right)=$ $P\left(k_{2}\right) g\left(\omega_{2}\right)$, with $P\left(k_{1}\right)$ and $P\left(k_{2}\right)$ being the degree distributions of layer I and II, respectively.

Panels (a) and (b) of Fig. 4 report the solutions of Eqs. (10) and (11), respectively. In both cases, it is easy to notice the presence of an unstable middle branch, which is responsible for the hysteretic loop associated to ES, and observed in Figs. 1 and 2.

Our analytic results allow a better and deeper understanding of the intimate causes for ES, and in particular of the microscopic mechanisms that are at the basis of the arousal of explosiveness in the transition. Indeed, if one considers the usual Kuramoto model $\dot{\theta}_{i}=\omega_{i}+\lambda \sum_{j=1}^{N} A_{i j} \sin \left(\theta_{j}-\theta_{i}\right)$ for the common second-order phase transition, and develops the same mean-field treatment, one obtains that the formula for the order parameter is

$$
R=\frac{1}{\langle k\rangle} \int_{|\omega| \leq \lambda R k} h(k, \omega) k \sqrt{1-\left(\frac{\omega}{\lambda R k}\right)^{2}} d \omega d k .
$$

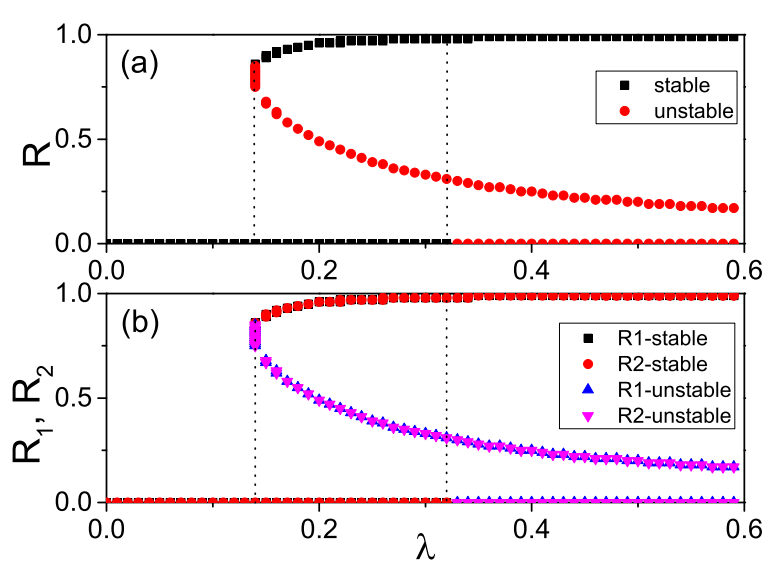

FIG. 4: (color online). Analytical solutions for the order parameter $R$. (a) $R$ vs. $\lambda$ for the single ER network of Eq. (1) with $f=1 . R$ is here calculated from Eq. 10, and parameters are the same as those in Fig. 1 (b) $R$ vs. $\lambda$ for the two-layered ER network of Eqs. (4) with $f=1$. $R_{1}$ ("squares" and "up triangles") and $R_{2}$ ("circles" and "down triangles") are evaluated from Eqs. (11) and the parameters are the same as in Fig. 2 a). In all cases, "dotted lines" are just guides for the eye.

Now, a distinctive difference between Eq. (12) and Eq. (10) is that the integration range $|\omega| \leq \lambda R k$ in Eq. (12) is replaced by $|\omega| \leq \lambda R^{2} k$ in Eq. (10). Such a replacement results actually in the following, remarkable, consequence. For the backward curves in Figs. 1 and 2 one has $R \approx R^{2} \approx 1$, and thus the difference between $R$ and $R^{2}$ is not so large. Instead, for the forward curves of the transition, one has $R \approx 0$ and thus the difference between $R$ and $R^{2}$ is there significant, i.e. $R^{2}$ will be much smaller than $R$. Notice, further, that the integration domains in Eqs. (12) and (10) actually determine the fraction of oscillators belonging to the main synchronization cluster. In other words, the larger synchronized clusters are forbidden to be formed in Eq. (10), in analogy with the suppressive rule recently discussed in Ref. [13]. In details, in the usual case of a second-order transition, the oscillators with closer natural frequencies will first form small synchronized clusters, and then these clusters will gradually grow up and merge with the increase of the coupling strength, up to eventually forming a giant cluster. On the contrary, in the present case, the factor $R^{2}$ in the integration domain has the effect of actually suppressing the merging of small synchronized clusters. Thus, with the increase of $\lambda$, more and more free oscillators will be attracted to the distinct clusters, but these clusters are prevented from merging each other. Eventually, when no more free oscillators are left, a discontinuous and abrupt behavior of $R$ will show up as the consequence of the sudden collapse of all clusters, determining a first-order like transition.

Notice that the above discussions holds for the case $f=$ 1. When $f<1$, the oscillators can actually be divided into two groups, the controlled fraction $f$ and the free fraction $1-$ $f$. The oscillators in the controlled fraction have a behavior 
similar to that of the case $f=1$, while those in the fraction $1-f$ will behave similarly to Eq. (12). Considering that the controlled group $f$ and the free group $1-f$ are in fact inter-connected, the behavior of the free part $1-f$ will be influenced by the controlled part $f$, implying that the merging of small clusters in the free part $1-f$ will be again suppressed. When $1>f>f_{c}$, this suppression mechanism will be the leading one, and therefore the system will exhibit ES. On the contrary, when $f<f_{c}$, the suppression mechanism will not be strong enough, and the merging behavior of small clusters in the free part $1-f$ will cause the occurrence of a secondorder smooth transition.

The most remarkable conclusion of our study is therefore that ES has, indeed, a microscopic root, but this root is essentially to be found in a microscopic mechanism able to suppress the formation of a giant synchronization cluster. While a positive correlation between the oscillators' natural frequencies and their degrees [6, 7] or couplings strength [9-11] has the effect of suppressing at all the formation of any synchronization cluster, in the present case (i.e. in the absence of any specific correlation features), the network nodes are initially able to form small independent synchronized clusters, each one of them being able to further grow with the increase of the coupling strength, and the suppression mechanism acts instead by impeding a merging process of the clusters. We cannot therefore exclude that even other forms of realizing such a suppressive rule, originating from yet unrevealed microscopic sources, would also determine the arousal of ES.

In conclusion, we reported on a novel framework for the setting of abrupt, explosive and irreversible transitions to synchronization in adaptive and multilayer networks, where it is possible to observe ES even without the requirement of positive correlations between natural frequencies and effective couplings of the networks' nodes. Our results are fully robust against large variations in the network topologies and frequency distributions. Based on these findings and in contrast with the accepted state of knowledge on the subject, we can safely conclude that the necessary condition for ES is, in fact, the existence of any microscopic suppressive rule able to prevent (in a way or in another) the formation of a giant synchronization cluster.

Work partially supported by the NNSF of China under Grant No. 11135001 and 11375066, Joriss project under Grant No. 78230050, 973 Program under Grant No. 2013CB834100, the Innovation Program of Shanghai Mu- nicipal Education Commission grant No. 12ZZ043, and the Open Project Program of State Key Laboratory of Theoretical Physics, Institute of Theoretical Physics, Chinese Academy of Sciences, China (No. Y4KF151CJ1).

* Electronic address: stefano.boccaletti@gmail.com

$\dagger$ Electronic address: zhliu@ phy.ecnu.edu.cn

[1] S. Boccaletti, V. Latora, Y. Moreno, M. Chavez, and D.-U. Hwang, Phys. Rep. 424, 175 (2006).

[2] B. M. Adhikari, C. M. Epstein, and M. Dhamala, Phys. Rev. E 88, 030701(R) (2013).

[3] S. V. Buldyrev, R. Parshani, G. Paul, H. E. Stanley, and S. Havlin, Nature 464, 1025 (2010).

[4] B. A. Huberman and R. M. Lukose, Science 277, 535 (1997).

[5] D. Pazó, Phys. Rev. E 72, 046211 (2005).

[6] J. Gómez-Gardeñes, S. Gómez, A. Arenas and Y. Moreno, Phys. Rev. Lett. 106, 128701 (2011).

[7] I. Leyva, R. Sevilla-Escoboza, J. M. Buldu, I. Sendina-Nadal, J. Gomez-Gardenes, A. Arenas, Y. Moreno, S. Gomez, R. JaimesReategui, and S. Boccaletti, Phys. Rev. Lett. 108, 168702 (2012).

[8] T. K. D. M. Peron and F. A. Rodrigues, Phys. Rev. E 86, 056108 (2012); ibid, 86, 016102 (2012); B. C. Coutinho, A. V. Goltsev, S. N. Dorogovtsev, and J. F. F. Mendes, Phys. Rev. E 87, 032106 (2013); W. Liu, Y. Wu, J. Xiao, and M. Zhan, Europhys. Lett. 101, 38002 (2013); P. Ji, T. K. DM. Peron, P. J. Menck, F. A. Rodrigues, and J. Kurths, Phys. Rev. Lett. 110, 218701 (2013).

[9] I. Leyva, I. Sendina-Nadal, J. A. Almendral, A. Navas, S. Olmi, and S. Boccaletti, Phys. Rev. E 88, 042808 (2013).

[10] X. Zhang, X. Hu, J. Kurths, and Z. Liu, Phys. Rev. E 88, 010802 (R) (2013).

[11] I. Leyva, A. Navas, I. Sendina-Nadal, J. A. Almendral, J. M. Buldu, M. Zanin, D. Papo, and S. Boccaletti, Sci. Rep. 3, 1281 (2013).

[12] P. Li, K. Zhang, X. Xu, J. Zhang, and M. Small, Phys. Rev. E 87, 042803 (2013); L. Zhu, L. Tian, and D. Shi, Phys. Rev. E 88, 042921 (2013).

[13] X. Zhang, Y. Zou, S. Boccaletti, and Z. Liu, Sci. Rep. 4, 5200 (2014).

[14] The stationary value of $\mathrm{R}$ is calculated by taking a long time average after the transient.

[15] R. Albert and A.-L. Barabási, Rev. Mod. Phys.74, 47 (2002).

[16] R. Parshani, S. V. Buldyrev, and S. Havlin, Proc. Natl. Acad. Sci.108, 1007 (2011).

[17] P. C. Matthews, R. E. Mirollo and S. H. Strogatz, Physica D 52, 293 (1991).

[18] P. S. Skardal and A. Arenas, Phys. Rev. E 89, 062811 (2014). 